\title{
Analysis of seizure EEG in kindled epileptic rats
}

\author{
A. K. SEN $\dagger^{*}$, M. J. KUBEK $¥$ and H. E. SHANNONף \\ $\dagger$ Department of Mathematical Sciences, Indiana University, Indianapolis, IN 46202, USA \\ $\ddagger$ Department of Anatomy and Cell Biology, Indiana University School of Medicine, Indianapolis, IN 46202, USA \\ ๆ Division of CNS Research, Eli Lilly and Company, Lilly Corporate Center, Indianapolis, IN 46285, USA
}

(Received 11 August 2006; revised 16 May 2007; in final form 20 June 2007)

\begin{abstract}
Using wavelet analysis we have detected the presence of chirps in seizure EEG signals recorded from kindled epileptic rats. Seizures were induced by electrical stimulation of the amygdala and the EEG signals recorded from the amygdala were analyzed using a continuous wavelet transform. A time-frequency representation of the wavelet power spectrum revealed that during seizure the EEG signal is characterized by a chirp-like waveform whose frequency changes with time from the onset of seizure to its completion. Similar chirp-like timefrequency profiles have been observed in newborn and adult patients undergoing epileptic seizures. The global wavelet spectrum depicting the variation of power with frequency showed two dominant frequencies with the largest amounts of power during seizure. Our results indicate that a kindling paradigm in rats can be used as an animal model of human temporal lobe epilepsy to detect seizures by identifying chirp-like time-frequency variations in the EEG signal.
\end{abstract}

Keywords: Seizure; EEG; Chirp; Kindling; Wavelet analysis

\section{Introduction}

Epilepsy is a neurological disorder that affects more than $1 \%$ of people worldwide. Temporal lobe epilepsy (TLE) is the most common type of epilepsy in humans. Many of the seizures that occur in patients with TLE are classified as complex partial seizures because they originate from and are confined within the temporal lobe. However, in nearly $60 \%$ of these patients, the seizures spread from the temporal lobe to other brain regions-a process referred to as secondary generalization. Many TLE patients have a history of brain injury such as febrile seizures or trauma. After recovering from the initial precipitating injury, these patients undergo a seizure-free latent period. The duration of the latent period varies substantially, ranging from a few days to decades, with an average of 6-9 years. Following this latent period, spontaneous recurrent seizures develop, which typically continue throughout life.

Epilepsy and seizures have been studied extensively through the use of laboratory animal models. Animal models are useful, particularly from a pharmacological point of view, i.e. for the development of antiepileptic drugs. More than 100 different types of animal models have been reported in the literature [1-7]. Among them, the following three have enjoyed the most popularity among researchers: (a) kindling model, (b) Kainate model and (c) pilocarpine model. Kindling, originally described by Goddard [8], is a well-established

\footnotetext{
*Corresponding author. Email: asen@iupui.edu
} 
procedure to induce seizures in animals [9-15]. Kindled seizures develop in response to focal, repeated, subconvulsive electrical stimulations of a specific limbic structure. Initial stimulations produce no overt behavioral changes, although the appearance of an epileptiform afterdischarge is necessary and sufficient for kindling to ensue. Upon successive stimulations, behavioral modifications develop, initially as facial twitching, followed by forelimb clonus and culminating in generalized convulsions of the tonic-clonic type [10,11]. Concurrent with these behavioral changes there is an increase in amplitude of the afterdischarge, and an appearance of seizure in regions which receive no direct stimulation. Once kindled, the brain remains permanently excitable and subsequent stimulations typically lead to seizures. Kindling results in a number of synaptic changes in the brain [16]. It also alters the expression of the immediate early genes [17] and several neuropeptides such as the thyrotropin-releasing hormone [18]. Various neurophysiological and pharmacological aspects of kindling are discussed in a recent review [19].

Kindled seizures are characterized as complex partial seizures with secondary generalization consisting of five behavioral stages: stages 1 and 2 of partial seizures manifested with chewing and head bobbing, stage 3 with forelimb clonus corresponding to partial or secondary generalized seizures, followed by stage 4 with rearing and stage 5 with loss of balance and falling - these last two stages being part of secondary generalized seizures $[11,20]$.

As an alternative to kindling, seizures can be induced by administering chemical convulsants such as kainate or pliocarpine. Kainate is an ionotropic glutamate receptor agonist, whereas pilocarpine is a muscarinic acetylcholine receptor agonist. In these models, there is an initial episode of status epilepticus which is followed by a seizure-free latent period and then by a state of spontaneous recurrent seizures. The kainate and pilocarpine models are referred to as Status Epilepticus (SE) models [21-29]. There are many similarities and differences among these three models with relative advantages and disadvantages [30]. Both kindling and SE models are known to produce epileptic seizures by two mechanisms: by excessive activation in pathways utilizing glutamate as the excitatory neurotransmitter and/or decreased inhibition in GABAergic pathways [31,32]. As described above, kindling seizures are evoked whereas the kainate and pilocarpine induced seizures are spontaneous. There are also differences in behavioral manifestations [33]. A wet dog shake is a characteristic feature of kainate and pliocarpine seizures, but it does not occur in kindled seizures. There is neuron loss and brain damage associated with the kainate and pilocarpine models, but not with kindling. Furthermore, unlike kindling, the SE models have a high mortality rate, particularly during status epilepticus.

The pharmacology of elicited kindled seizures is remarkably similar to the pharmacology of spontaneous seizures that occur in the SE models. In other words, kindled seizures respond to the same antiepileptic drugs that block spontaneous seizures in SE models. However, when these models are used to study the antiepileptogenic effects of drugs, major differences between the kindling and SE models exist, even among different SE models [34,35]. It should also be pointed out that many humans with TLE do not have status epilepticus as their initial seizure event and this may be a limitation of the SE models. However, the SE models can reliably produce the latent period and spontaneous seizures found in humans. Both kindling and SE models suffer from the disadvantage that they have age and strain specificity; as a consequence, it is sometimes difficult to generalize the findings of a specific animal model [36,37].

As an experimental model of human epilepsy, the kindling paradigm offers several procedural advantages. In kindling, precise focal activation of target brain sites is possible, development of chronic epileptogenesis can be readily observed, the patterns of seizure propagation and generalization can be carefully monitored, and interictal, ictal and postictal 
periods can be easily manipulated [38,39]. Some of the disadvantages of kindling are: kindling experiments are labor intensive, and spontaneous seizures do not develop unless the animals are given a very large number of stimulations (on the order of hundreds), i.e. unless the animals are over-kindled [40-42].

One of the neuropathological characteristics of human TLE is hippocampal sclerosis, which is typically associated with neuronal damage in amygdala, hippocampus and entorhinal cortex. Interestingly, the SE animal models such as the kainate and pilocarpine models show a similar neuropathology but the kindled models do not. No hippocampal damage is found to occur even when kindled rats develop spontaneous recurrent seizures as the result of many amygdala stimulations [41].

A characteristic feature of human TLE is that during seizure the frequency of the EEG signal changes with time. A signal with time-varying frequency is referred to as a chirp signal. Using intracranial recordings from different brain regions of epileptic patients, several investigators observed the presence of chirps in the EEG signal during seizure [43-47]. In particular, Schiff et al. [45] found that chirps are very sensitive detectors of seizures and are highly specific as markers (with no false positive detections). They proposed that chirps in EEG signals can be used as a spectral signature of epileptic seizures in humans. In the present work we examine a kindled rat model and show that the seizure EEG signal exhibits a chirp-like time-frequency pattern similar to that observed in humans. Analogous to the situation in humans, we shall find that in the kindled rats, the EEG frequency during seizure first increases with time followed by a gradual decrease with the progression of seizure.

The dynamics of seizures in epileptic patients and animal models are usually studied by recording the EEG signals from depth electrodes inserted into the brain or from scalp electrodes. The EEG signals are typically nonstationary in nature, i.e. their mean and/or variance do not remain constant in time [47]. Due to nonstationarity, these signals are not amenable to analysis by the traditional spectral methods such as the Fourier transform. A Fourier transform determines the frequency content of a signal through spectral peaks but it loses all the temporal information in the signal. An efficient way to analyze these nonstationary signals and determine their time-varying spectrum is to use wavelet-based techniques [48]. Wavelets have been used in diverse applications including the analysis of seizures in humans (for example, $[49,50]$ ).

In an earlier work, Gath et al. [51] studied generalized seizures with focal onset in (a) a kainate induced rat model, (b) patients with TLE. Using multivariate autoregressive modeling with adaptive segmentation of the EEG signal, they estimated the variation in signal power with both time and frequency. They found that during a seizure event, the timefrequency profile has a chirp-like characteristic with an initial rise in frequency followed by a gradual decrease, both in the kainate model and in epileptic patients.

Here we study the dynamics of seizure activity in kindled epileptic rats using wavelet analysis. Seizures were induced by electrical stimulation of the amygdala and the EEG signals recorded from the amygdala were analyzed using a continuous wavelet transform (CWT) [48]. A time-frequency representation of the wavelet power spectrum reveals the chirp-like character of the EEG signal during seizure. The global wavelet spectrum depicting the variation of time-averaged power with frequency shows two dominant frequencies with the largest amounts of power during seizure. To our knowledge, wavelet-based analysis of seizure EEG in kindled animals has not been reported in the literature. Presence of chirps in seizure EEG signals recorded from kindled rats has also not been observed earlier.

The presentation in this paper is organized as follows. First we describe the experimental procedure and data acquisition methods. This is followed by an analysis of the seizure EEG 
signals using a CWT. Finally we demonstrate the presence of chirps from the results of the wavelet analysis.

\section{Methods}

\subsection{Experimental procedure}

As mentioned in the introduction, we used electrical stimulation to induce seizure activity in the rats. Briefly, male Sprauge Dawley rats were anesthetized with isoflurane and bipolar stainless steel electrodes $(0.25 \mathrm{~mm}$ diameter; Plastics One, Roanoke, VA) were stereotaxically implanted bilaterally into the basolateral amygdala for stimulation and recording. After electrode implantation, the animals were allowed at least one week to recover from surgery before beginning the stimulation and recording phases of the experiments.

During recording the rats were housed in a clear plexiglass chamber and the electrodes were connected through a commutator (model SL3C, Plastics One) to a Model 15RX Physiodata Amplifier System (Grass Instruments Division, Astro-Med, Inc., Warwick, RI). Evoked afterdischarge threshold testing was completed and only those animals eliciting afterdischarge were used for subsequent kindling. Qualified subjects were stimulated once every $24 \mathrm{~h}$ using $1.0 \mathrm{~s}$ train of $60 \mathrm{~Hz}$ biphasic square wave pulses of $0.1 \mathrm{~ms}$ duration and $200 \mu$ A peak-to-peak amplitude. Behavioral seizures were scored according to the method of Racine $[10,11]$. The duration of clonus and evoked ipsilateral and contralateral afterdischarge durations were recorded following each stimulation. This procedure took $8 \pm 2$ days for electrical kindling in the rats to reach initial stage 5 seizure. After five consecutive daily stage 5 seizures have occurred, the animals were considered fully (permanently) kindled. Permanence is defined as a fully kindled animal responding to a subconvulsive stimulus that results in a stepwise stereotypy (stages 1 through 4) and becomes generalized (stage 5) following weeks to several months of being unstimulated. We routinely tested for kindling permanence 20 days following the initial kindling criteria by giving 1-3 subthreshold $(200 \mu \mathrm{A})$ stimulations and recording afterdischarge and clonus duration. The data were digitized and recorded using PolyView software (Grass Instruments; Astro-Med, Inc.) and stored for subsequent analysis.

\subsection{Wavelet analysis of seizure EEG signal}

A wavelet is a small wave with a compact support. In order to be classified as a wavelet, a function $\psi(t)$ should have zero mean and finite energy:

$$
\int_{-\infty}^{\infty} \psi(t) \mathrm{d} t=0, \quad \int_{-\infty}^{\infty}|\psi(t)|^{2} \mathrm{~d} t<\infty .
$$

The CWT of a function $x(t)$ with respect to a wavelet $\psi(t)$ is defined as a convolution of the function with a scaled and translated version of $\psi(t)$. The wavelet $\psi(t)$ is referred to as an analyzing wavelet or a mother wavelet. The convolution is expressed by the integral [48]:

$$
W(s, \tau)=\int_{-\infty}^{\infty} x(t) \psi_{s, \tau}^{*}(t) \mathrm{d} t,
$$


where

$$
\psi_{s, \tau}(t)=\frac{1}{\sqrt{s}} \psi\left(\frac{t-\tau}{s}\right)
$$

is a scaled and translated version of the mother wavelet $\psi(t)$ and an asterisk on $\psi$ denotes its complex conjugate. The symbols $s$ and $\tau$ are called a scale parameter and a translation parameter, respectively. The scale parameter controls the dilation $(s>1)$ and contraction $(s<1)$ of the wavelet, whereas the translation parameter indicates the location of the wavelet in time. The factor $1 / \sqrt{s}$ is introduced in equation (3) so that the function $\psi_{s, \tau}(t)$ has unit energy at every scale. The wavelet power spectrum of the signal representing the energy at scale $s$ is defined as the squared modulus of the CWT:

$$
P(s, \tau)=|W(s, \tau)|^{2} .
$$

The integral formulation for a CWT as given in equation (2) applies to a signal $x(t)$ that is a continuous function. In order to use it for a discrete signal given by the time series such as the EEG signal, this integral representation must be discretized in an appropriate fashion. Consider a discrete time series $\left\{x_{\mathrm{n}}\right\}$ with $n=1,2,3, \ldots, N$. For such a time series, equation (2) may be discretized as [51]:

$$
W_{n}(s)=\sum_{n^{\prime}=1}^{N}\left(\frac{\delta \tau}{s}\right)^{1 / 2} x_{n^{\prime}} \psi^{*}\left[\frac{\left(n^{\prime}-n\right) \delta \tau}{s}\right]
$$

Here $n$ is the time index, and $\delta \tau$ is the sampling interval. The factor $(\delta \tau / s)^{1 / 2}$ preserves the unit energy property referred to earlier. In order to calculate the CWT using equation (5), the convolution given by this equation should be performed $N$ times for each scale. However, as shown in [52], it is possible to carry out all $N$ convolutions simultaneously in Fourier space by means of a discrete Fourier transform (DFT).

Using equation (5), the wavelet power spectrum for each scale can be evaluated as: $\left|W_{n}(s)\right|^{2}$. This power spectrum which depends on both scale and time is represented by a surface. By taking contours of this surface and plotting them on a plane, a time-scale representation of the wavelet power spectrum may be obtained. Alternatively, the results may be presented on a time-frequency plane by using the scale-to-frequency conversion formula: $f=f_{0} f_{s} / s$ where $f$ is the frequency corresponding to the scale $s, f_{0}$ is the center frequency of the mother wavelet and $f_{s}$ is the sampling frequency. In our analysis we have used a complex Morlet wavelet as the mother wavelet. A Morlet wavelet consists of a plane wave modulated by a Gaussian function and is described by [48]:

$$
\psi(\eta)=\pi^{-1 / 4} e^{i \omega_{0} \eta} e^{-\eta^{2} / 2}
$$

where $\omega_{0}=2 \pi f_{0}$, with $f_{0}$ being the center frequency. Strictly speaking, the Morlet wavelet as given in equation (6) is not a wavelet since it does not satisfy the property of zero mean. The zero-mean property can be satisfied by introducing a correction factor [48]. However, for $\omega_{0}>5$, this correction factor is negligible and then equation (6) can be used without loss of accuracy. Note that the coefficient $\pi^{-1 / 4}$ is used as a normalization factor to ensure that the Morlet wavelet has unit energy. Morlet wavelets have been used successfully in a wide variety of applications for feature extraction in time series data including neurophysiological applications [53]. 


\section{Results and discussion}

Figure 1 depicts a representative time series of the EEG signal recorded from a depth electrode implanted in the amygdala. The stimulus artifact has been removed from the signal and the trace in figure 1 begins immediately following the stimulus artifact. This time series is obtained by sampling the raw EEG data at $200 \mathrm{~Hz}$.

We have applied a CWT on this time series using a Morlet wavelet with $\omega_{0}=6$ as the mother wavelet. This value of $\omega_{0}$ provides a good balance between time and frequency localizations. A time-frequency representation of the resulting wavelet power spectrum is obtained as a contour plot. We also computed the global wavelet spectrum of the EEG signal by averaging the wavelet power spectrum over the entire time interval. This is given by:

$$
\bar{W}^{2}(s)=\frac{1}{N} \sum_{n=1}^{N}\left|W_{n}(s)^{2}\right|
$$

The global wavelet spectrum is similar to a smoothed Fourier spectrum which can be computed with a fast Fourier transform [52].

In figure 2, we have shown a time-frequency representation of the wavelet power spectrum of the EEG time series depicted in figure 1. The colors blue and red represent highest and lowest power levels, respectively. This figure reveals a chirp-like frequency variation of the seizure EEG signal with time. In particular, we find that the frequency changes with time from the onset of seizure to its completion. It is also apparent from figure 2 that during the initial phase of the seizure the frequency increases with time followed by a gradual decrease as time progresses. A similar time-frequency profile has been recently reported by Dikanev et al. [47] and others in adult patients undergoing generalized seizure.

The global wavelet spectrum of the seizure EEG signal is shown in figure 3. This figure has two spectral peaks revealing the two dominant frequencies at approximately 4.2 and $2.2 \mathrm{~Hz}$, corresponding to the largest amounts of power.

Our results indicate that a kindling paradigm in rats can be used as an animal model of human TLE from the standpoint of time-frequency variations. There are several possible explanations for the origin of chirps in seizure EEG signals. These have been discussed in [45]. As mentioned in the introduction, a kainate induced rat seizure model was analyzed by Gath et al. [48], who also observed the presence of chirps in the seizure EEG signal. Note that in a kindling model, seizures are evoked, whereas the kainate or pilocarpine model involves

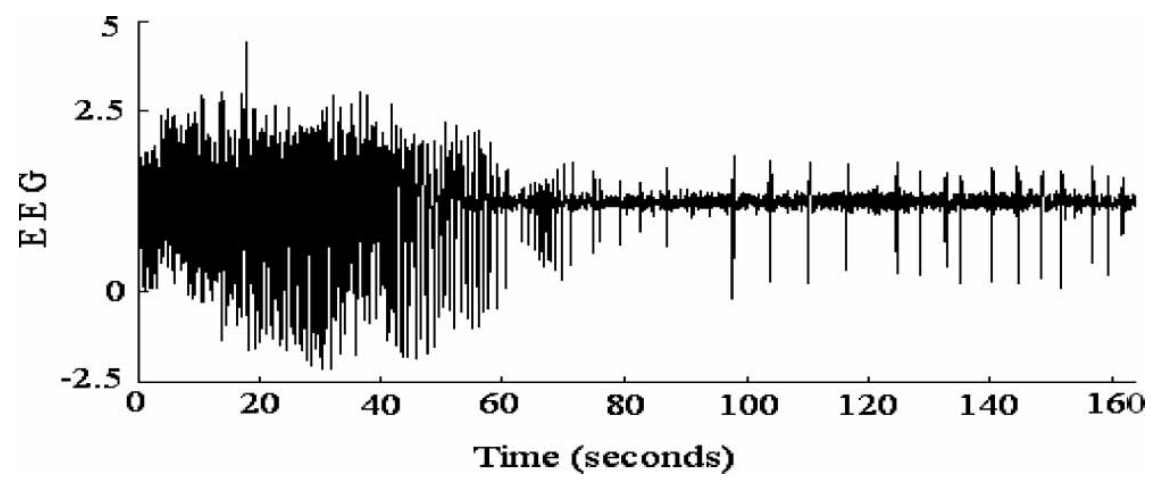

Figure 1. A time series of the EEG signal recorded from the amygdala. The EEG magnitudes are in arbitrary units. 


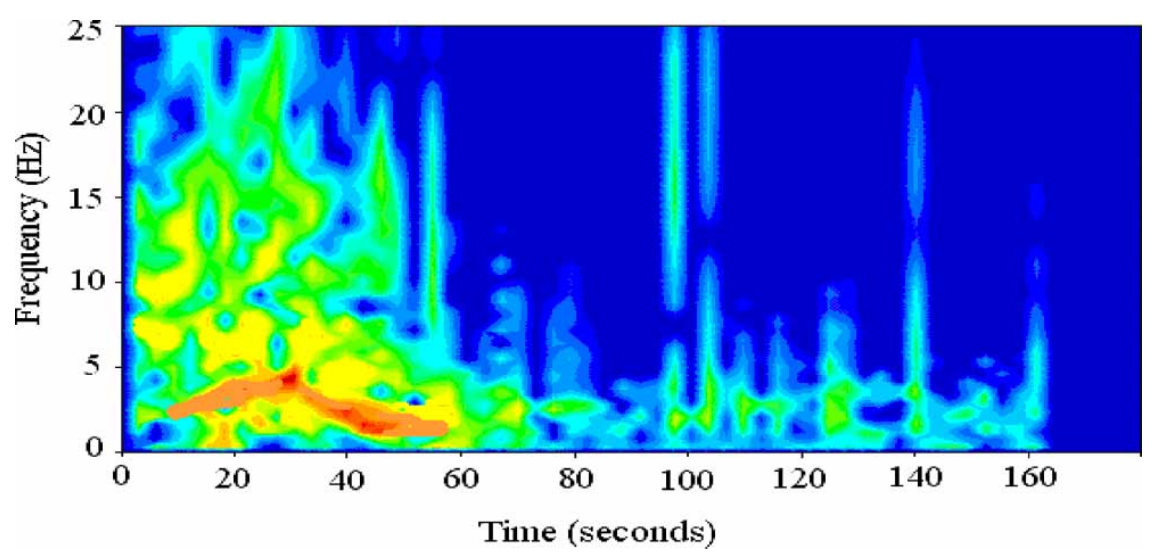

Figure 2. A time-frequency representation of the wavelet power spectrum of the EEG signal shown in figure 1, obtained by a CWT. A Morlet mother wavelet of order 6 has been used.

an initial episode of status epilepticus followed by a latent period and subsequent spontaneous recurrent seizures. Although seizures are induced in a different manner in these two types of models, the chirp-like time-frequency patterns in both models are similar to what is observed in humans with TLE.

\section{Concluding remarks}

Currently there is a great deal of interest in developing efficient methods to predict and prevent the onset of seizures in epileptic patients [54-60]. Some of these methods detect seizures by identifying chirps in the time-frequency landscape of the EEG signal using wavelets. Our results indicate that analogous to humans, a seizure event in a kindled rat model is characterized by the presence of chirps in the EEG signal. Thus a kindled animal model may be used effectively to test the different seizure prediction methods. As a laboratory technique, kindling offers the researcher rigorous experimental control and allows experimental protocols to be tested in a systematic manner. In this regard, as well as from the

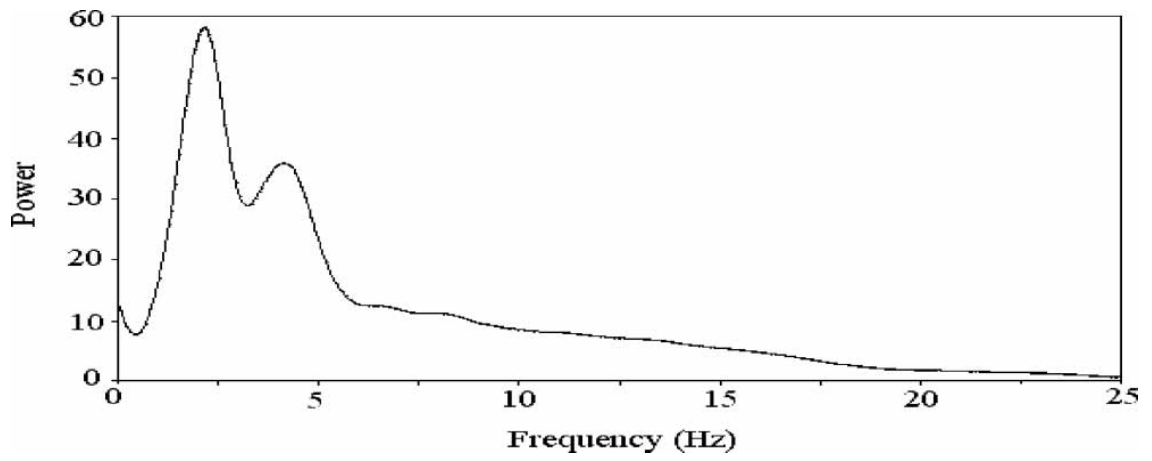

Figure 3. Global wavelet spectrum of the EEG signal depicted in figure 1. This plot shows the variation of power with frequency revealing two dominant frequencies during seizure. The power is in arbitrary units. 
standpoint of drug discovery, the kindling paradigm will continue to serve as an investigative model of human epilepsy and seizures.

\section{References}

[1] Fisher, R., 1989, Animal models of epilepsies, Brain Research, 14, 245-278.

[2] Löscher, W., 1997, Animal models of intractable epilepsy, Progress in Neurobiology, 53, 239-258.

[3] Coulter, D.A., McIntyre, D.C. and Löscher, W., 2002, Animal models of limbic epilepsies: what can they tell us?, Brain Pathology, 12, 240-256.

[4] Cole, A.J., Koh, S. and Zhang, Y., 2002, Are seizures harmful: what can we learn from animal models?, Progress in Brain Research, 135, 13-23.

[5] Jefferys, J.G.R., 2003, Models and mechanisms of experimental epilepsies, Epilepsia, 44(suppl.12), 44-50.

[6] Buckmaster, P.S., 2004, Laboratory animal models of temporal lobe epilepsy, Comparative Medicine, 54, 473-485.

[7] Schwartzkroin, P.A. and Engel, J., 2006, What good are animal models? In: A. Pitkänen, P.A. Schwartzkroin and S.L. Moshé (Eds.) Models of Seizures and Epilepsy (NewYork: Elsevier), pp. 659-668.

[8] Goddard, G., 1967, The development of epileptic seizures through brain stimulation at low intensity, Nature, 214, 1020-1021.

[9] Goddard, G.V., McIntyre, D.C. and Leech, C., 1969, A permanent change in brain function resulting from daily electrical stimulation, Experimental Neurology, 25, 295-330.

[10] Racine, R.J., 1972, Modification of seizure activity by electrical stimulation: I. after-discharge threshold, Electroencephalography and Clinical Neurophysiology, 32, 269-279.

[11] Racine, R.J., 1972, Modification of seizure activity by electrical stimulation: II. Motor seizure, Electroencephalography and Clinical Neurophysiology, 32, 281-294.

[12] Kelly, M.E., 1998, The kindling model of temporal lobe epilepsy. In: S.L. Peterson and T.E. Albertson (Eds.) Neuropharmacolgy Methods in Epilepsy Research (Boca Raton: CRC Press), pp. 41-75.

[13] Nissinen, J., Halonen, T., Koivisto, E. and Pitkänen, A., 2000, A new model of temporal lobe epilepsy induced by electrical stimulation of amygdala in rat, Epilepsy Research, 38, 177-205.

[14] McIntyre, D.C., Poulter, M.O. and Gilby, K., 2002, Kindling: some old and some new, Epilepsy Research, 50, $79-92$.

[15] McIntyre, D.C., 2006, The kindling phenomenon. In: A. Pitkänen, P.A. Schwartzkroin and S.L. Moshé (Eds.) Models of Seizures and Epilepsy (NewYork: Elsevier), pp. 351-363.

[16] Sutula, T.P., 1990, Experimental models of temporal lobe epilepsy: new insights from the study of kindling and synaptic reorganization, Epilepsia, 31, S45-S54.

[17] Simonato, M.G., Hosford, D.A., Labiner, D.M., Shin, C., Mansbach, H.H. and McNamara, M., 1991, Differential expression of immediate early genes in the hippocampus in the kindling model of epilepsy, Molecular Brain Research, 11, 115-124.

[18] Knoblach, S.M. and Kubek, M.J., 1997, Increases in thyrotropin-releasing hormone messenger RNA expression induced by a model of human temporal lobe epilepsy: effect of partial and complete kindling, Neuroscience, 76, 85-95.

[19] Corcoran, M.E. and Moshé, S.L. (Eds.), 2004, Kindling 6. Advances in Behavioral Biology (NewYork: Springer-Verlag), p. 55

[20] Gastaut, H., 1970, Clinical and electroencephalographic classifications of epileptic seizures, Epilepsia, 11, $102-113$.

[21] Turski, L., Ikonomidou, C. and Turski, W.A., 1989, Review: cholinergic mechanisms and epileptogenesis: the seizure induced by pilocarpine: a novel model of intractable epilepsy, Synapse, 3, 154-171.

[22] Sperk, G., 1994, Kainic acid seizures in the rat, Progress in Neurobiology, 42, 1-32.

[23] Cavalheiro, E.A., Santos, N.F. and Priel, M.R., 1996, The pilocarpine model of epilepsy in mice, Epilepsia, 37, 1015-1019.

[24] Hellier, J.L., Patrylo, P.R., Buckmaster, P.S. and Dudek, F.E., 1998, Recurrent spontaneous motor seizures after repeated low-dose systemic treatment with kainite: assessment of a rat model of temporal lobe epilepsy, Epilepsy Research, 31, 79-84.

[25] Bragin, A., Engel, J., Wilson, C.L., Vizentin, E. and Mathern, G.W., 1999, Electrophysiologic analysis of a chronic seizure model after unilateral hippocampal KA injection, Epilepsia, 40, 1210-1221.

[26] Leite, J.P., Garcia-Cairasco, N. and Cavalheiro, E.A., 2002, New insights from the use of pilocarpine and kainite models, Epilepsy Research, 50, 93-103.

[27] Furtado, M.D.A., Braga, G.K., Oliveira, J.A.C., Vecchio, F.D. and Garcia-Cairasco, N., 2002, Behavioral, morphologic and electroencephalographic evaluation of seizures induced by intrahippocampal microinjection of pilocarpine, Epilepsia, $\mathbf{4 3}$ (suppl. 5), 37-39.

[28] Dudek, F.E., Clark, S., Williams, P.A. and Grabenstatter, H.L., 2006, Kainate-induced status epilepticus: a chronic model of acquired epilepsy. In: A. Pitkänen, P.A. Schwartzkroin and S.L. Moshé (Eds.) Models of Seizures and Epilepsy (NewYork: Elsevier), pp. 415-430. 
[29] Cavalheiro, E.A., Naffah-Mazzacoratti, M.G., Mello, L.E. and Leite, J.P., 2006, The pilocarpine model of seizures. In: A. Pitkänen, P.A. Schwartzkroin and S.L. Moshé (Eds.) Models of Seizures and Epilepsy (NewYork: Elsevier), pp. 433-444.

[30] Morimoto, K., Fahnestock, M. and Racine, R.J., 2004, Kindling and status epilepticus models of epilepsy: rewiring the brain, Progress in Neurobiology, 73, 1-60.

[31] Morimoto, K., 1989, Seizure-triggering mechanisms in the kindling model of epilepsy: collapse of GABAmediated inhibition and activation of NMDA receptors, Neuroscience and Biobehavioral Reviews, 13, 253-260.

[32] Treiman, D.M., 2001, GABAergic mechanisms in epilepsy, Epilepsia, 42, 8-12.

[33] Veliškova, J., 2006, Behavioral characterization of seizures in rats. In: A. Pitkänen, P.A. Schwartzkroin and S.L. Moshé (Eds.) Models of Seizures and Epilepsy (NewYork: Elsevier), pp. 601-610.

[34] Löscher, W., 2002, Animal models of epilepsy for development of antiepilepto-genic and disease-modifying drugs. A comparison of the pharmacology of kindling and post-status epilepticus models of temporal lobe epilepsy, Epilepsy Research, 50, 105-123.

[35] Löscher, W., 2002, Current status and future directions in the pharmacology of epilepsy, Trends in Pharmacological Sciences, 23, 113-118.

[36] Golden, G.T., Smith, G.G., Ferraro, T.N. and Reyes, P.F., 1995, Rat strain and age differences in kainic acid induced seizures, Epilepsy Research, 20, 151-159.

[37] Schauwecker, P.E., 2002, Complications associated with genetic background effects in models of experimental epilepsy, Progress in Brain Research, 135, 139-148.

[38] Sato, M., Racine, R.J. and McIntyre, D.C., 1990, Kindling: basic mechanisms and clinical validity, Electroencephalography and Clinical Neurophysiology, 76, 459-472.

[39] Morimoto, K., Sato, H., Osawa, M. and Sato, M., 1998, Contributions of kindling to clinical epileptology. In: M.E. Corcoran and S.L. Moshe (Eds.) Kindling 5 (NewYork: Plenum Press), pp. 485-494.

[40] Michalakis, M., Holsinger, D., Ikeda-Douglas, C., Cammisuli, S., Forbinteau, J., et al., 1998, Development of spontaneous seizures over extended electrical kindling I. Electrographic, behavioral and transfer kindling correlates, Brain Research, 793, 197-211.

[41] Tuunanen, J. and Pitkänen, A., 2000, Do seizures cause neuronal damage in rat amygdala kindling?, Epilepsy Research, 39, 171-176.

[42] Sutula, T.P. and Ockuly, J., 2006, Kindling, spontaneous seizures and the consequences of epilepsy: more than a model. In: A. Pitkänen, P.A. Schwartzkroin and S.L. Moshé (Eds.) Models of Seizures and Epilepsy, pp. 395-406.

[43] Franaszczuk, P.J., Bergey, G.K., Durka, P.J. and Eisenberg, H.M., 1998, Time-frequency analysis using the matching pursuit algorithm applied to seizures originating from the mesial temporal lobe, Electroencephalography and Clinical Neurophysiology, 106, 512-521.

[44] Wendling, F., Shamsollahi, M.B., Badier, J.M. and Bellanger, J.J., 1999, Time-frequency matching of warped depth-EEG seizure observations, IEEE Transactions on Bio-Medical Engineering, 46, 601-605.

[45] Schiff, S.J., Colella, D., Jacyna, G.M., Hughes, E., Creekmore, J.W., Marshall, A., Bozek-Kuzmicki, M., Benke, G., Gaillard, W.D., Conry, J. and Weinstein, S.R., 2000, Brain chirps: spectrographic signatures of epileptic seizures, Clinical Neurophysiology, 111, 953-958.

[46] Boashash, B. and Mesbah, M., 2001, A time-frequency approach for newborn seizure detection, IEEE Engineering Medicine and Biology, 20, 133-143.

[47] Dikanev, T., Smirnov, D., Wennberg, R., Perez Velazquez, J.L. and Bezruchko, B., 2005, EEG nonstationarity during intracranially recorded seizures: statistical and dynamical analysis, Clinical Neurophysiology, 116, $1796-1807$.

[48] Addison, P.S., 2002, The Illustrated Wavelet Transform Handbook (Philadelphia: Institute of Physics Publishing).

[49] Rosso, O.A., Blanco, S. and Rabinowicz, A., 2003, Wavelet analysis of generalized tonic-clonic epileptic seizures, Signal Processing, 83, 1275-1289.

[50] Adeli, H., Zhou, Z. and Dadmehr, N., 2003, Analysis of EEG records in an epileptic patient using wavelet transform, Journal of Neuroscience Methods, 123, 69-87.

[51] Gath, I., Feuerstein, C., Pham, D.T. and Rondouin, G., 1992, On the tracking of rapid dynamic changes in seizure EEG, IEEE Transactions on Bio-Medical Engineering, 39, 952-958.

[52] Torrence, C. and Compo, G.P., 1998, A practical guide to wavelet analysis, Bulletin of American meteorological Society, 79, 61-78.

[53] Samar, V.J., Bopardikar, A., Rao, R. and Swartz, K., 1999, Wavelet analysis of neuroelectric waveforms: a conceptual tutorial, Brain and Language, 66, 7-60.

[54] Osorio, I., Frei, M.G. and Wilkinson, S.B., 1998, Real-time automated detection and quantitative analysis of seizures and short-term prediction of clinical onset, Epilepsia, 39, 615-627.

[55] Geva, A.B. and Kerem, D.H., 1998, Forecasting generalized epileptic seizures from the EEG signal by wavelet analysis and dynamic unsupervised fuzzy clustering, IEEE Transactions on Bio-Medical Engineering, 45, 1205-1216. 
[56] Ouyang, G., Li, X. and Guan, X., 2006 Application of wavelet-based similarity analysis to epileptic seizure prediction, Computers in Biology and Medicine, in press.

[57] Mizuno-Matsumoto, Y., Motamedi, G.K., Webber, W.R.S. and Lesser, R.P., 2002, Wavelet cross-correlation analysis can help predict whether bursts of pulse stimulation will terminate afterdischarges, Clinical Neurophysiology, 113, 33-42.

[58] Khan, Y.U. and Gotman, J., 2003, Wavelet-based automatic seizure detection in intracereberal electroencephalogram, Clinical Neurophysiology, 114, 898-908.

[59] Subasi, A., 2005, Epileptic seizure detection using dynamic wavelet network, Expert Systems with Applications, 29, 343-355.

[60] Niederhauser, J., Esteller, R., Echauz, J., Vachtsevanos, G. and Litt, B., 2003, Detection of seizure precursors from depth-EEG using a sign periodogram transform, IEEE Transactions on Bio-Medical Engineering, 51, 449-458. 


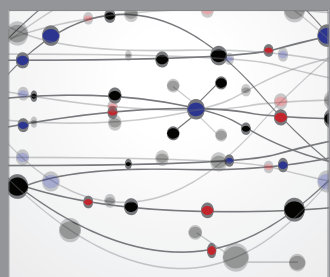

The Scientific World Journal
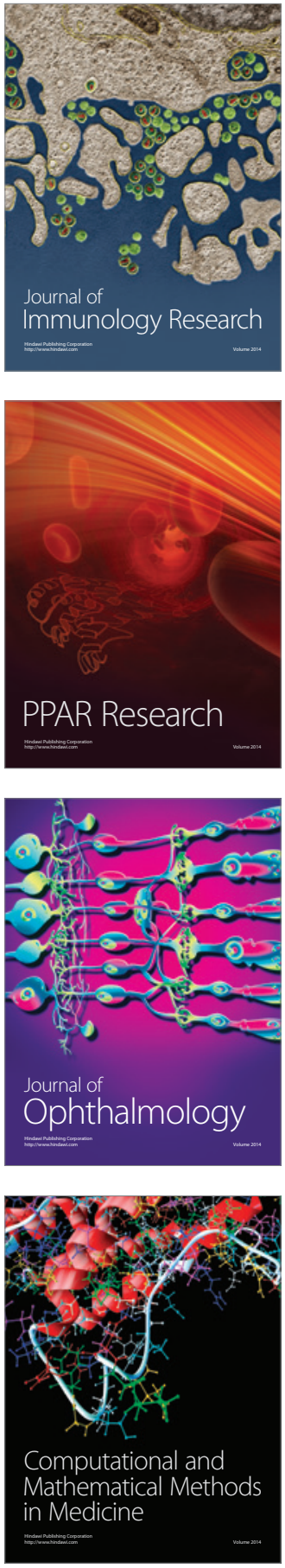

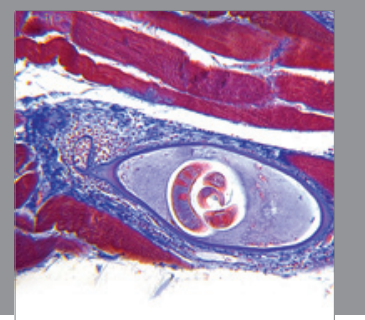

Gastroenterology

Research and Practice
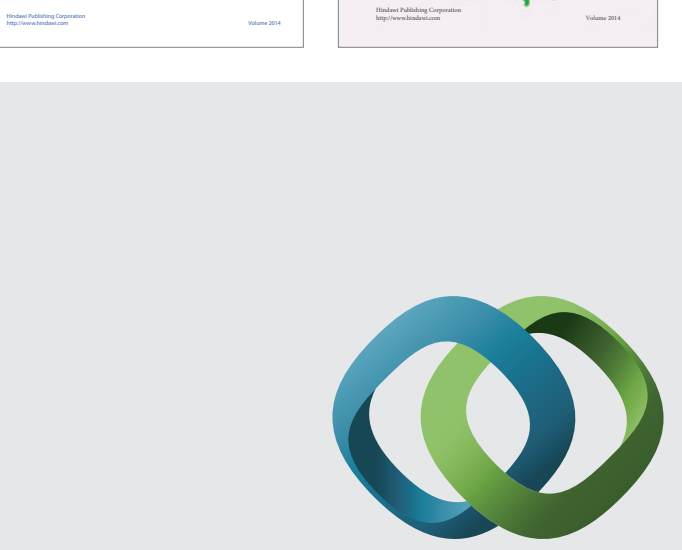

\section{Hindawi}

Submit your manuscripts at

http://www.hindawi.com
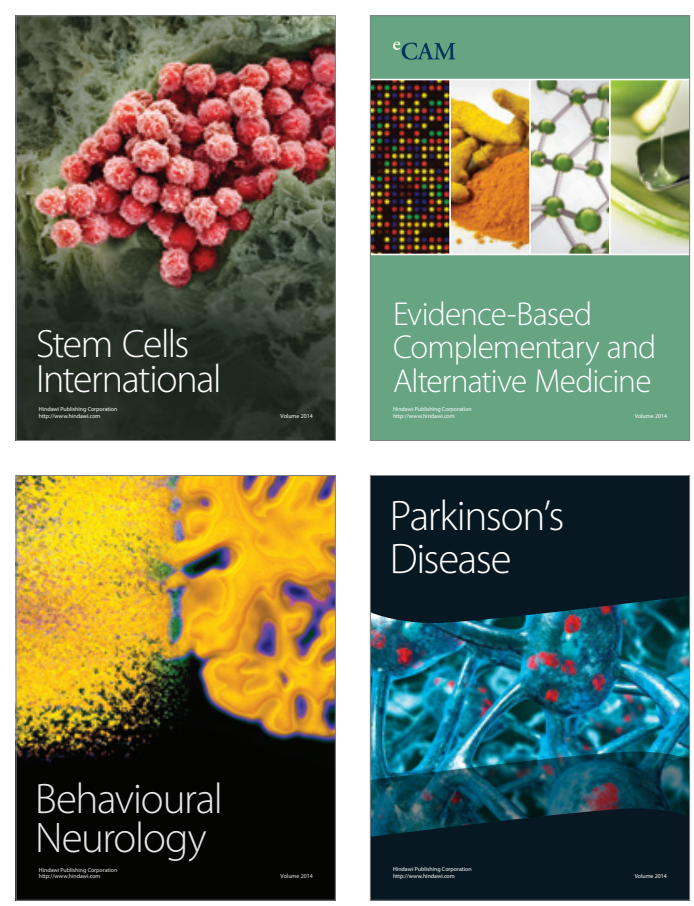

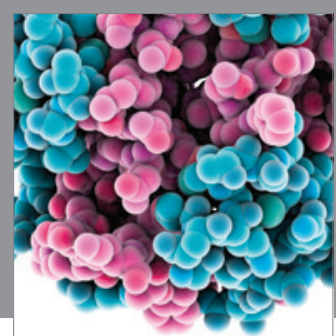

Journal of
Diabetes Research

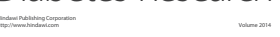

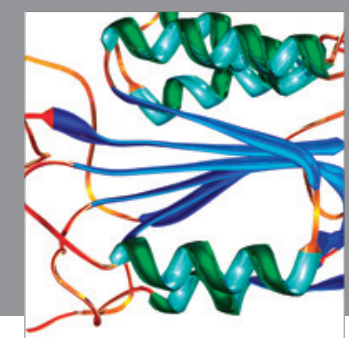

Disease Markers
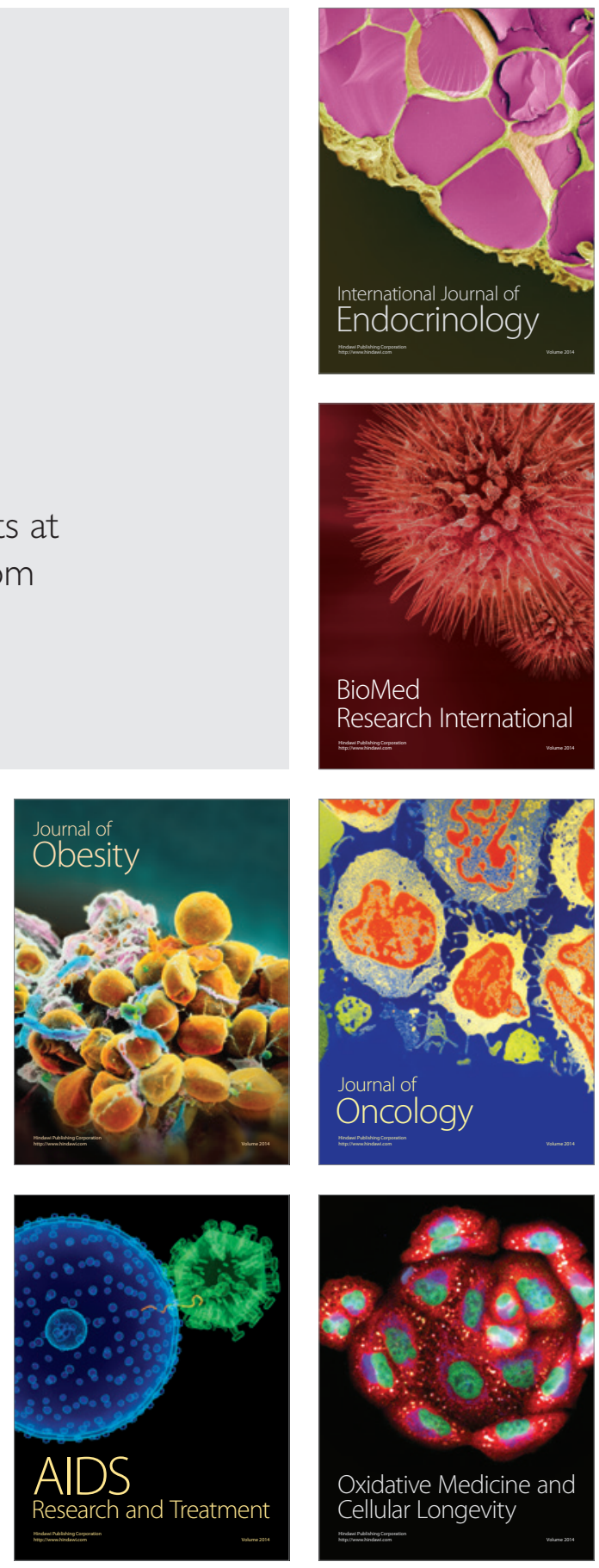